\title{
Subsequence Sums of Zero-sum-free Sequences
}

\author{
Pingzhi Yuan \\ School of Mathematics, South China Normal University, \\ Guangzhou 510631, P. R. CHINA \\ mcsypz@mail.sysu.edu.cn
}

Submitted: Apr 25, 2009; Accepted: Jul 30, 2009; Published: Aug 7, 2009

Mathematics Subject Classification: 11B75, 11B50

\begin{abstract}
Let $G$ be a finite abelian group, and let $S$ be a sequence of elements in $G$. Let $f(S)$ denote the number of elements in $G$ which can be expressed as the sum over a nonempty subsequence of $S$. In this paper, we slightly improve some results of [10] on $f(S)$ and we show that for every zero-sum-free sequences $S$ over $G$ of length $|S|=\exp (G)+2$ satisfying $f(S) \geqslant 4 \exp (G)-1$.
\end{abstract}

Key words: Zero-sum problems, Davenport's constant, zero-sum-free sequence.

\section{Introduction}

Let $G$ be a finite abelian group (written additively)throughout the present paper. $\mathcal{F}(G)$ denotes the free abelian monoid with basis $G$, the elements of which are called sequences (in $G$ ). A sequence of not necessarily distinct elements from $G$ will be written in the form $S=g_{1} \cdot \cdots \cdot g_{n}=\prod_{i=1}^{n} g_{i}=\prod_{g \in G} g^{v_{g}(S)} \in \mathcal{F}(G)$, where $\mathrm{v}_{g}(S) \geqslant 0$ is called the multiplicity of $g$ in $S$. Denote by $|S|=n$ the number of elements in $S$ (or the length of $S)$ and let $\operatorname{supp}(S)=\left\{g \in G: \mathrm{v}_{g}(S)>0\right\}$ be the support of $S$.

We say that $S$ contains some $g \in G$ if $\mathrm{v}_{g}(S) \geqslant 1$ and a sequence $T \in \mathcal{F}(G)$ is a subsequence of $S$ if $\mathrm{v}_{g}(T) \leqslant \mathrm{v}_{g}(S)$ for every $g \in G$, denoted by $T \mid S$. If $T \mid S$, then let $S T^{-1}$ denote the sequence obtained by deleting the terms of $T$ from $S$. Furthermore, by $\sigma(S)$ we denote the sum of $S$, (i.e. $\left.\sigma(S)=\sum_{i=1}^{k} g_{i}=\sum_{g \in G} \vee_{g}(S) g \in G\right)$. By $\sum(S)$ we denote the set consisting of all elements which can be expressed as a sum over a nonempty subsequence of $S$, i.e.

$$
\sum(S)=\{\sigma(T): T \text { is a nonempty subsequence of } S\} .
$$

Supported by the Guangdong Provincial Natural Science Foundation (No. 8151027501000114) and NSF of China (No. 10571180). 
We write $f(S)=\left|\sum(S)\right|,\langle S\rangle$ for the subgroup of $G$ generated by all the elements of $S$.

Let $S$ be a sequence in $G$. We call $S$ a zero-sum sequence if $\sigma(S)=0$, a zero-sumfree sequence if $\sigma(W) \neq 0$ for any subsequence $W$ of $S$, and squarefree if $v_{g}(S) \leqslant 1$ for every $g \in G$.

Let $D(G)$ be the Davenport's constant of $G$, i.e., the smallest integer $d$ such that every sequence $S$ of elements in $G$ with $|S| \geqslant d$ satisfies $0 \in \sum(S)$. For every positive integer $r$ in the interval $\{1, \ldots, D(G)-1\}$, let

$$
f_{G}(r)=\min _{S,|S|=r} f(S),
$$

where $S$ runs over all zero-sumfree sequences of $r$ elements in $G$.

In 1972, Eggleton and Erdös (see [4]) first tackled the problem of determining the minimal cardinality of $\sum(S)$ for squarefree zero-sum-free sequences (that is for zero-sumfree subsets of $G$ ). In 2006, Gao and Leader [5] proved the following result.

Theorem A [5] Let $G$ be a finite abelian group of exponent $m$. Then

(i) If $1 \leqslant r \leqslant m-1$ then $f_{G}(r)=r$.

(ii) If $\operatorname{gcd}(6, m)=1$ and $G$ is not cyclic then $f_{G}(m)=2 m-1$.

In 2007, Sun[11] showed that $f_{G}(m)=2 m-1$ still holds without the restriction that $\operatorname{gcd}(6, m)=1$.

Using some techniques from the author [12], the author [13] proved the following two theorems.

Theorem $\mathbf{B}([9],[13])$ Let $S$ be a zero-sumfree sequence in $G$ such that $\langle S\rangle$ is not a cyclic group, then $f(S) \geqslant 2|S|-1$.

Theorem C ([13]) Let $S$ be a zero-sumfree sequence in $G$ such that $\langle S\rangle$ is not a cyclic group and $f(S)=2|S|-1$. Then $S$ is one of the following forms

(i) $S=a^{x}(a+g)^{y}, x \geqslant y \geqslant 1$, where $g$ is an element of order 2 .

(ii) $S=a^{x}(a+g)^{y} g, x \geqslant y \geqslant 1$, where $g$ is an element of order 2.

(iii) $S=a^{x} b, x \geqslant 1$.

However, Theorem B is an old theorem of Olson and White (see [10] Theorem 1.5) which has been overlooked by the author.

Recently, by an elegant argument, Pixton [10] proved the following result.

Theorem D ([10]) Let $G$ be a finite abelian group and $S$ a zero-sum-free sequence of length $n$ generating a subgroup of rank greater than 2, then $f(S) \geqslant 4|S|-5$.

One purpose of the paper is to slightly improve the above result of Pixton. We have

Theorem 1.1 Let $n \geqslant 2$ be a positive integer. Let $G$ be a finite abelian group and $S=\left(g_{i}\right)_{i=1}^{n}$ a zero-sum-free sequence of length $n$ generating a subgroup $H$ of rank 2 and $H \nRightarrow C_{2} \oplus C_{2 m}$, where $m$ is a positive integer. Suppose that

$$
\sum(S) \neq A_{a} \cup\left(b+B_{a}\right)
$$

where $a, b \in G, A_{a}, B_{a}$ are some subsets of the cyclic group $\langle a\rangle$ generated by a and $b \notin\langle a\rangle$, then $f(S) \geqslant 3 n-4$. 
Theorem 1.2 Let $n \geqslant 5$ be a positive integer. Let $G$ be a finite abelian group and $S=\left(g_{i}\right)_{i=1}^{n}$ a zero-sum-free sequence of length $n$ generating a subgroup $H$ of rank 2 and $H \nRightarrow C_{2} \oplus C_{2 m}, \approx C_{3} \oplus C_{3 m}, \approx C_{4} \oplus C_{4 m}$, where $m$ is a positive integer. Suppose that

$$
\sum(S) \neq A_{a} \cup\left(b+B_{a}\right), A_{a} \cup\left(b+B_{a}\right) \cup\left(2 b+C_{a}\right), A_{a} \cup\left(b+B_{a}\right) \cup\left(-b+C_{a}\right),
$$

where $a, b \in G, A_{a}, B_{a}, C_{a}$ are some subsets of the cyclic group $\langle a\rangle$ generated by a and $b \notin\langle a\rangle$, then $f(S) \geqslant 4 n-9$.

Theorem 1.3 Let $G$ be an abelian group and $S=\left(g_{i}\right)_{i=1}^{n}$ is a zero-sum-free sequence of length $n \geqslant 5$ that generating a subgroup of rank greater than 2 and $\langle S\rangle \nRightarrow C_{2} \oplus C_{2} \oplus C_{2 m}$, then $f(S)|\geqslant 4| S \mid-3$ except when $S=a^{x}(a+g)^{y} c, a^{x}(a+g)^{y} g c, a^{x} b c$, where $a, b, c, g$ are elements of $G$ with ord $(g)=2$, in these cases, $f(S)=4|S|-5$ when the rank of the subgroup generated by $S$ is 3 .

Another main result of the paper runs as follows.

Theorem 1.4 Let $G=C_{n_{1}} \oplus \ldots \oplus C_{n_{r}}$ be a finite abelian group with $1<n_{1}|\ldots| n_{r}$. If $r \geqslant 2$ and $n_{r-1} \geqslant 4$, then every zero-sum-free sequence $S$ over $G$ of length $|S|=n_{r}+2$ satisfies $f(S) \geqslant 4 n_{r}-1$.

This partly confirms a former conjecture of Bollobás and Leader [2] and a conjecture of Gao, Li, Peng and Sun [6], which is outlined in Section 5.

The paper is organized as follows. In Section 2 we present some results on Davenport's constant. In section 3 we prove more preliminary results which will be used in the proof of the main Theorems. The proofs of Theorems 1.1 to 1.3 are given in Section 4 . In section 5 we will prove Theorem 1.4 and give some applications of Theorems 1.1 and 1.2.

\section{Some bounds on Davenport's constant}

Lemma 2.1 (see [8]) Let $G$ be a non-cyclic finite abelian group. Then $D(G) \leqslant \frac{|G|}{2}+1$.

Lemma 2.2 ([10] Lemma 4.1) Let $k \in \mathbb{N}$. If $H \leqslant G$ are some finite abelian groups and $G_{1}=G / H \simeq(\mathbb{Z} / 2 \mathbb{Z})^{k+1}$. Then $D(G) \leqslant 2 D(H)+2^{k+1}-2$.

Lemma 2.3 ([10] Lemma 2.3) Let $H \leqslant G$ be some finite abelian groups and $G_{1}=G / H$ is non-cyclic, then $D(G) \leqslant\left(D\left(G_{1}\right)-1\right) D(H)+1$.

Lemma 2.4 (i)Let $G$ be a finite abelian group of rank 2 and $G \neq C_{2} \oplus C_{2 m}$. Then (i) $D(G) \leqslant \frac{|G|}{3}+2$.

(ii) $D\left((\mathbb{Z} / p \mathbb{Z})^{r}\right)=r(p-1)+1$ for prime $p$ and $r \geqslant 1$.

(iii) $D(G) \leqslant|G|$.

Proof. (iii) is obvious. (i) and (ii) follow from Theorems 5.5.9 and 5.8.3 in [7]. 
Lemma 2.5 If $G$ is an abelian group of rank greater than 2 and $G \neq C_{2} \oplus C_{2} \oplus C_{2 m}$, then $D(G) \leqslant \frac{|G|+2}{4}$.

Proof. Since $G$ has rank greater than 2, then $G$ has $p$-rank at least 3 for some prime $p$, and thus there exists a subgroup $H \leqslant G$ with $G / H \simeq(\mathbb{Z} / p \mathbb{Z})^{3}$. We can then apply Lemmas 2.3 and 2.4 (ii),(iii) to conclude that

$$
D(G) \leqslant \frac{3(p-1)}{p^{3}}|G|+1 \leqslant \frac{2}{9}|G|+1 \leqslant \frac{|G|+2}{4}
$$

when $p \geqslant 3$. If $p=2$ we can apply Lemmas 2.1 and 2.2 to see that

$$
D(G) \leqslant 2 D(H)+6 \leqslant 2 \cdot\left(\frac{|H|}{2}+1\right)+6=\frac{|G|}{8}+8 \leqslant \frac{|G|+2}{4}
$$

when $|G| \geqslant 60$. Further, the only case with $|G| \leqslant 60$ and $G \neq C_{2} \oplus C_{2} \oplus C_{2 m}$ is that $G \cong C_{2} \oplus C_{4} \oplus C_{4}$, in this case $D(G)=8 \leqslant \frac{32+2}{4}$. We are done.

Lemma 2.6 ([10] Theorem 5.3) If $G$ is an abelian group of rank greater than 2, and let $X \subseteq G \backslash\{0\}$ be a generating set for $G$ consisting only of elements of order greater than 2. Suppose $A \subset G$ satisfies $|(A+x) \backslash A| \leqslant 3$ for all $x \in X$. Then $\min \{|A|,|G \backslash A|\} \leqslant 5$.

Lemma 2.7 ([10] Lemma 4.3) Let $G$ be a finite abelian group and let $X \subseteq G \backslash\{0\}$ be a generating set for $G$. Suppose $A$ is a nonempty proper subset of $G$. Then

$$
\sum_{x \in X}|(A+x) \backslash A| \geqslant|X| .
$$

Lemma 2.8 ([10] Lemma 4.4) Let $G$ be a finite abelian group and let $X \subseteq G \backslash\{0\}$ be a generating set for $G$. Suppose $f: G \rightarrow \mathbb{Z}$ is a function on $G$. Then

$$
\sum_{x \in X g \in G} \max \{f(g+x)-f(g), 0\} \geqslant(\max (f)-\min (f))|X| .
$$

Using the technique in the proof of [10] Theorem 5.3, we have

Lemma 2.9 Let $m>0$ be a positive integer and $G$ a finite abelian group, and let $X \subseteq$ $G \backslash\{0\}$ be a generating set for $G$. Suppose $A \subseteq G$ satisfies $|(A+x) \backslash A| \leqslant m$ for all $x \in X$ and there exists a proper subset $Y \subset X$ such that $H=\langle Y\rangle$ and $G_{1}=G / H$ both contain at least $(m+1)$ elements. Then $\min \{|A|,|G \backslash A|\} \leqslant m^{2}$.

Proof. First, without loss of generality we may replace $X$ by a minimal subset $X_{1}$ of $X$ such that $\left\langle X_{1} \cap Y\right\rangle=\langle Y\rangle$ and $\left\langle X_{1}\right\rangle=G$. 
Define a function $f: G_{1} \rightarrow \mathbb{Z}$ by $f(g)=|A \cap(g+H)|$. Then we have that

$$
\begin{aligned}
|(A-x) \backslash A| & =\sum_{g \in G_{1}}|((A-x) \backslash A) \cap(g+H)| \\
& =\sum_{g \in G_{1}}|(A-x) \cap(g+H)|-|(A-x) \cap A \cap(g+H)| \\
& =\sum_{g \in G_{1}}|(A) \cap(g+x+H)|-|(A-x) \cap A \cap(g+H)| \\
& \geqslant \sum_{g \in G_{1}} \max \{f(g+x)-f(g), 0\} .
\end{aligned}
$$

It follows that

$$
\begin{aligned}
m|X \backslash Y| & \geqslant \sum_{x \in X \backslash Y}|(A-x) \backslash A| \\
& \geqslant \sum_{x \in X \backslash Y} \sum_{g \in G_{1}} \max \{f(g+x)-f(g), 0\} \\
& \geqslant(\max (f)-\min (f))|X \backslash Y|
\end{aligned}
$$

by Lemma 2.8, since $X \backslash Y$ projects to $|X \backslash Y|$ distinct nonzero elements in $G_{1}$ because $X$ is a minimal generating set with the property described in the first paragraph. Thus $(\max (f)-\min (f)) \leqslant m$. Then by replacing $A$ by $G \backslash A$ if necessary, we can assume that $f(g) \neq|H|$ for any $g \in G_{1}$. The reason is that

$$
(G \backslash A+x) \backslash(G \backslash A)=A \backslash(A+x),
$$

So

$$
|(G \backslash A+x) \backslash(G \backslash A)|=|A \backslash(A+x)|=|(A-x) \backslash A| .
$$

Since for every $x \in Y$ we have

$$
\begin{aligned}
|(A+x) \backslash A| & =\sum_{g \in G_{1}}|((A+x) \backslash A) \cap(g+H)| \\
& =\sum_{g \in G_{1}} \mid((A+x) \cap(g+H)-(A+x) \cap A \cap(g+H) \mid \\
& =\sum_{g \in G_{1}} \mid((A+x) \cap(g+H+x)-((A+x) \cap(g+x+H)) \cap(A \cap(g+H)) \mid \\
& =\sum_{g \in G_{1}} \mid((A \cap(g+H))+x-(A \cap(g+H)+x) \cap(A \cap(a+H)) \mid \\
& =\sum_{g \in G_{1}} \mid((A \cap(g+H)+x) \backslash(A \cap(g+H)) \mid,
\end{aligned}
$$


thus we can apply Lemma 2.7 to obtain that

$$
\begin{aligned}
m|Y| & \geqslant \sum_{x \in Y}|(A+x) \backslash A| \\
& =\sum_{g \in G_{1}} \sum_{x \in Y} \mid((A \cap(g+H)+x) \backslash(A \cap(g+H)) \mid \\
& \geqslant|\operatorname{supp}(f)||Y|,
\end{aligned}
$$

where $\operatorname{supp}(f)=\left\{g \in G_{1} \mid f(g) \neq 0\right\}$ is the support of $f$. Since $\left|G_{1}\right| \geqslant m+1$, this implies that $f(g)=0$ for some $g$, and thus $f(g) \leqslant m$ for all $g \in G_{1}$. Then $|A|=\sum_{g \in G_{1}} f(g) \leqslant$ $\max (f)|\operatorname{supp}(f)| \leqslant m^{2}$, as desired.

\section{$3 \quad$ Proof of Theorems 1.1 to 1.3}

Proof of Theorem 1.1:

Proof. We first prove the theorem if $S$ contains an element of order 2. Suppose that $S=\left(g_{i}\right)_{i=1}^{n}$ generates $G, G$ has rank $2,0 \notin \sum(S)$, and $g_{n}$ has order 2 . Let $\bar{G}$ be the quotient of $G$ by the subgroup generated by $g_{n}$, then $\bar{G}$ has rank 2 since $G \neq C_{2} \oplus C_{2 m}$. Let $\bar{S}=\left(\bar{g}_{i}\right)_{i=1}^{n-1}$ be the projection of the first $n-1$ terms of $S$ to $\bar{G}$. Then $0 \in \sum(\bar{S})$ would imply that either 0 or $g_{n}$ lies in $\sum\left(\left(g_{i}\right)_{i=1}^{n-1}\right)$ and hence $0 \in \sum(S)$, so $\left\langle\left(g_{i}\right)_{i=1}^{n-1}\right\rangle$ is not a cyclic group and $\sum(S)=\sum\left(\left(g_{i}\right)_{i=1}^{n-1}\right) \cup\left\{g_{n}\right\} \cup\left(\sum\left(\left(g_{i}\right)_{i=1}^{n-1}\right)+g_{n}\right)$ is a disjoint union. Therefore, by Theorem B

$$
f(S) \geqslant 2 f\left(\left(g_{i}\right)_{i=1}^{n-1}\right)+1 \geqslant 2(2 n-3)+1 \geqslant 4 n-5 \geqslant 3 n-4,
$$

as desired.

Now suppose for contradiction that the theorem fails for some abelian group $G$ of minimum size. Choose $S=\left(g_{i}\right)_{i=1}^{n}$ to be a counterexample sequence of minimum length $n$, so $f(S) \leqslant 3 n-5$. Also, $S$ must generate $G$ by the minimality of $|G|$, so $G$ is noncyclic, $G \nRightarrow C_{2} \oplus C_{2 m}$. Moreover, by the minimality of $n$ we have that either the theorem holds for all $S g_{i}^{-1}(1 \leqslant i \leqslant n)$; or $\left\langle S g_{i}^{-1}\right\rangle \cong C_{2} \oplus C_{2 m}$, or $\sum\left(S g_{i}^{-1}\right)=A_{a} \cup\left(b+B_{a}\right)$, where $a, b \in G, A_{a}, B_{a}$ are some subsets of the cyclic group $\langle a\rangle$ generated by $a$ and $b \notin\langle a\rangle$ for some $1 \leqslant i \leqslant n$. We divide the remaining proof into three cases.

Case 1: $\left\langle S g_{i}^{-1}\right\rangle \cong C_{2} \oplus C_{2 m}$ for some $1 \leqslant i \leqslant n$. Then $S=\left(S g_{i}^{-1}\right) g_{i}$ and $g_{i} \notin\left\langle S g_{i}^{-1}\right\rangle$ since $G \neq C_{2} \oplus C_{2 m}$. It follows that $\sum(S)=\sum\left(S g_{i}^{-1}\right) \cup\left\{g_{i}\right\} \cup\left(\sum\left(S g_{i}^{-1}\right)+g_{i}\right)$ is a disjoint union, by Theorem B we have $f(S) \geqslant 2 f\left(S g_{i}^{-1}\right)+1 \geqslant 2(2 n-3)+1 \geqslant 3 n-4$, as desired.

Case 2: $\sum\left(S g_{i}^{-1}\right)=A_{a} \cup\left(b+B_{a}\right)$ for some $1 \leqslant i \leqslant n$. Then $g_{i} \notin\langle a\rangle$ since $\sum(S) \neq A_{a} \cup\left(b+B_{a}\right)$. By the definitions of $\sum\left(S g_{i}^{-1}\right)$, we have $S g_{i}^{-1}=S\left(g_{i} g_{j}\right)^{-1} g_{j}, g_{j}=$ $b+l a \notin\langle a\rangle, S\left(g_{i} g_{j}\right)^{-1} \subseteq\langle a\rangle$ and $j \neq i$. It follows that $\sum\left(S g_{i}^{-1}\right)=A_{a} \cup\left\{g_{j}\right\} \cup\left(g_{j}+A_{a}\right):=$ $A, A_{a} \subseteq\langle a\rangle$ is a disjoint union and

$$
\sum(S)=A \cup\left\{g_{i}\right\} \cup B, B=\left(g_{i}+A_{a}\right) \cup\left\{g_{i}+g_{j}\right\} \cup\left(g_{i}+g_{j}+A_{a}\right) .
$$


If $g_{i}=g_{j}$ or $A \cap B \neq \emptyset$, then $x_{i} \in(b+\langle a\rangle) \cup(-b+\langle a\rangle)$, and thus

$$
\sum(S)=A_{a} \cup\left(b+B_{a}\right) \cup\left(2 b+C_{a}\right), \quad \text { or } \quad A_{a} \cup\left(b+B_{a}\right) \cup\left(-b+C_{a}\right),
$$

where $A_{a}, B_{a}, C_{a}$ are some subsets of $\langle a\rangle$.

If $g_{i} \in b+\langle a\rangle$, then $g_{i}=b+k a$ for some $k \in \mathbb{Z}$ and

$$
\sum(S) \supset A_{a} \cup\left(b+B_{a}\right) \cup\left(2 b+k a+B_{a}\right),
$$

and the right hand side is a disjoint union, and thus

$$
f(S) \geqslant\left|A_{a}\right|+\left|B_{a}\right|+\left|B_{a}\right| \geqslant n-2+2(n-1)=3 n-4 .
$$

If $g_{i} \in-b+\langle a\rangle$, then $g_{i}=-b+k a$ for some $k \in \mathbb{Z}$ and

$$
\sum(S) \supseteq A_{a} \cup\left(b+B_{a}\right) \cup\left(-b+k a+\left(A_{a} \cup\{0\}\right)\right.
$$

and $A_{a} \cup\left(b+B_{a}\right) \cup\left(-b+k a+\left(A_{a} \cup\{0\}\right)\right.$ is a disjoint union, and thus

$$
f(S) \geqslant\left|A_{a}\right|+\left|B_{a}\right|+\left|A_{a}\right|+1 \geqslant n-2+2(n-1)=3 n-4 .
$$

If $g_{i} \neq g_{j}$ and $A \cap B=\emptyset$, then $\sum(S)=A \cup\left\{g_{i}\right\} \cup B, B=\left(g_{i}+A_{a}\right) \cup\left\{g_{i}+g_{j}\right\} \cup\left(g_{i}+g_{j}+A_{a}\right)$ is a disjoint union, hence

$$
f(S)=4\left|A_{a}\right|+3 \geqslant 4(n-2)+3 \geqslant 3 n-4 .
$$

Case 3: If the theorem holds for all $S g_{i}^{-1}, 1 \leqslant i \leqslant n$. Let $A=\sum(S) \subseteq G$. Then for any $i$ we have $\sum\left(S g_{i}^{-1}\right) \subseteq\left(A-g_{i}\right) \cap A$, so

$$
\left|\left(A-g_{i}\right) \backslash A\right| \leqslant f(S)-f\left(S g_{i}^{-1}\right) \leqslant 3 n-5-(3(n-1)-4)=2 .
$$

It is easy to see that $S$ satisfies the conditions of Lemma 2.9 since $\langle S\rangle \nsubseteq C_{2} \oplus C_{2 m}$. Applying Lemma 2.9 to $A \subseteq G$ with generating set $S$, we obtain that either $A$ or $G \backslash A$ has cardinality at most 4 . Since $|A|>4$, so we have that $|G \backslash A| \leqslant 4$.

We now consider the two cases. If $|G \backslash A|=1$, then $n \leqslant D(G)-1 \leqslant \frac{|G|}{3}+1$ by Lemma 2.4(i), and hence

$$
|G|=|A|+1 \leqslant 3 n-5+1 \leqslant|G|-1,
$$

which is a contradiction.

Otherwise, there is some nonzero element $y \in G \backslash A$, and $S$ is still zero-sum free after appending $-y$, so $n \leqslant D(G)-2 \leqslant \frac{|G|}{3}$ by Lemma 2.4(i) again, and thus

$$
|G| \leqslant|A|+4 \leqslant 3 n-5+4 \leqslant|G|-1,
$$

is again a contradiction. Theorem 1.1 is proved. 


\section{Proof of Theorem 1.2:}

Proof. For $|S|=5$, by Theorems 1.1, we have $f(S) \geqslant 3|S|-4=4|S|-9$, so the theorem holds for $n=5$. If $S=\left(g_{i}\right)_{i=1}^{n}$ contains an element of order 2, say, $o\left(g_{n}\right)=2$. By the similar argument as in Theorem 1.1 and by Theorem B, we have

$$
f(S) \geqslant 2 f\left(\left(g_{i}\right)_{i=1}^{n-1}\right)+1 \geqslant 2(2 n-3)+1 \geqslant 4 n-5,
$$

as desired.

Now suppose for contradiction that the theorem fails for some abelian group $G$ of minimum size. Choose $S=\left(g_{i}\right)_{i=1}^{n}$ to be a counterexample sequence of minimum length $n$, so $f(S) \leqslant 4 n-10$. Also, $S$ must generate $G$ by the minimality of $|G|$, so $G$ is noncyclic, $G \neq C_{2} \oplus C_{2 m}, ¥ C_{3} \oplus C_{3 m}, \neq C_{4} \oplus C_{4 m}$. Moreover, by the minimality of $n$ we have that either the theorem holds for all $S g_{i}^{-1}(1 \leqslant i \leqslant n)$, or $\left\langle S g_{i}^{-1}\right\rangle \cong C_{2} \oplus C_{2 m}$, or $\left\langle S g_{i}^{-1}\right\rangle \cong C_{3} \oplus C_{3 m}$, or $\left\langle S g_{i}^{-1}\right\rangle \cong C_{4} \oplus C_{4 m}$, or $\sum\left(S g_{i}^{-1}\right)=A_{a} \cup\left(b+B_{a}\right)$, or $A_{a} \cup\left(b+B_{a}\right) \cup\left(2 b+C_{a}\right)$, or $A_{a} \cup\left(b+B_{a}\right) \cup\left(-b+C_{a}\right)$, where $a, b \in G, A_{a}, B_{a}, C_{a}$ are some subsets of the cyclic group $\langle a\rangle$ generated by $a$ and $b \notin\langle a\rangle$ for some $1 \leqslant i \leqslant n$. We divide the remaining proof into five cases.

Case 1: $\left\langle S g_{i}^{-1}\right\rangle \cong C_{2} \oplus C_{2 m}$, or $\left\langle S g_{i}^{-1}\right\rangle \cong C_{3} \oplus C_{3 m}$ or $\left\langle S g_{i}^{-1}\right\rangle \cong C_{4} \oplus C_{4 m}$ for some $1 \leqslant i \leqslant n$. Then $S=\left(S g_{i}^{-1}\right) g_{i}$ and $g_{i} \notin\left\langle S g_{i}^{-1}\right\rangle$ since $G \neq C_{2} \oplus C_{2 m}, G \neq C_{3} \oplus C_{3 m}$ and $G \neq C_{4} \oplus C_{4 m}$. It follows that $\sum(S)=\sum\left(S g_{i}^{-1}\right) \cup\left\{g_{i}\right\} \cup\left(\sum\left(S g_{i}^{-1}\right)+g_{i}\right)$ is a disjoint union, by Theorem B we have $f(S) \geqslant 2 f\left(S g_{i}^{-1}\right)+1 \geqslant 2(2 n-3)+1 \geqslant 4 n-5$, as desired.

Case 2: $\sum\left(S g_{i}^{-1}\right)=A_{a} \cup\left(b+B_{a}\right)$ for some $1 \leqslant i \leqslant n$. Then $g_{i} \notin\langle a\rangle$ since $\sum(S) \neq A_{a} \cup\left(b+B_{a}\right)$. By the definitions of $\sum\left(S g_{i}^{-1}\right)$, we have $S g_{i}^{-1}=\left(S\left(g_{i} g_{i}\right)^{-1}\right) g_{j}, g_{j}=$ $b+l a \notin\langle a\rangle, S\left(g_{i} g_{j}\right)^{-1} \subseteq\langle a\rangle$ and $j \neq i$. It follows that $\sum\left(S g_{i}^{-1}\right)=A_{a} \cup\left\{g_{j}\right\} \cup\left(g_{j}+A_{a}\right):=$ $A, A_{a} \subseteq\langle a\rangle$ is a disjoint union and

$$
\sum(S)=A \cup\left\{g_{i}\right\} \cup B, B=\left(g_{i}+A_{a}\right) \cup\left\{g_{i}+g_{j}\right\} \cup\left(g_{i}+g_{j}+A_{a}\right) .
$$

If $g_{i}=g_{j}$ or $A \cap B \neq \emptyset$, then $g_{i} \in(b+\langle a\rangle) \cup(-b+\langle a\rangle)$, and thus

$$
\sum(S)=A_{a} \cup\left(b+B_{a}\right) \cup\left(2 b+C_{a}\right), \quad \text { or } \quad A_{a} \cup\left(b+B_{a}\right) \cup\left(-b+C_{a}\right),
$$

where $A_{a}, B_{a}, C_{a}$ are some subsets of $\langle a\rangle$, a contradiction. It follows that $\sum(S)=A \cup$ $\left\{g_{i}\right\} \cup B, B=\left(g_{i}+A_{a}\right) \cup\left\{g_{i}+g_{j}\right\} \cup\left(g_{i}+g_{j}+A_{a}\right)$ is a disjoint union, and thus $f(S)=$ $4\left|A_{a}\right|+3 \geqslant 4\left|S\left(g_{i} g_{j}\right)^{-1}\right|+3=4(n-2)+3=4 n-5$, as desired.

Case 3: $\sum\left(S g_{i}^{-1}\right)=A_{a} \cup\left(b+B_{a}\right) \cup\left(2 b+C_{a}\right):=A$ for some $1 \leqslant i \leqslant n$. Then $g_{i} \notin\langle a\rangle$ since $\sum(S) \neq A_{a} \cup\left(b+B_{a}\right) \cup\left(2 b+C_{a}\right)$. By the definitions of $\sum\left(S g_{i}^{-1}\right)$, we have $S g_{i}^{-1}=\left(S\left(g_{i} g_{j} g_{k}\right)^{-1}\right) g_{j} g_{k}, g_{j}=b+l a \notin\langle a\rangle, g_{k}=b+l_{1} a \notin\langle a\rangle,\left(S\left(g_{i} g_{j} g_{k}\right)^{-1}\right) \subseteq\langle a\rangle$ and $j \neq k \neq i$. It follows that $\sum\left(S g_{i}^{-1}\right)=A_{a} \cup\left(b+B_{a}\right) \cup\left(2 b+C_{a}\right):=A, A_{a} \subseteq\langle a\rangle$ is a disjoint union and $\left|A_{a}\right| \geqslant\left|S\left(g_{i} g_{j} g_{k}\right)^{-1}\right|=n-3,\left|B_{a}\right| \geqslant\left|A_{a}\right|+1 \geqslant n-2,\left|C_{a}\right| \geqslant\left|A_{a}\right|+1 \geqslant n-2$. And

$$
\sum(S)=A \cup\left\{g_{i}\right\} \cup B, B=\left(g_{i}+A\right) .
$$


If $g_{i}=g_{j}$ or $g_{i}=g_{k}$ or $A \cap B \neq \emptyset$, then $g_{i} \in(b+\langle a\rangle) \cup(-b+\langle a\rangle) \cup(2 b+\langle a\rangle) \cup(-2 b+\langle a\rangle)$ and $b$ is an element of order at least 4 by the assumptions. If $g_{i} \in b+\langle a\rangle$, then $g_{i}=b+k a$ for some $k \in \mathbb{Z}$ and

$$
\sum(S)=A_{a} \cup\left(b+B_{a}^{\prime}\right) \cup\left(2 b+C_{a}^{\prime}\right) \cup\left(3 b+k a+C_{a}\right), B_{a} \subseteq B_{a}^{\prime}, C_{a} \subseteq C_{a}^{\prime}
$$

is a disjoint union, and thus

$$
f(S)=\left|A_{a}\right|+\left|B_{a}^{\prime}\right|+\left|C_{a}\right|+\left|C_{a}\right| \geqslant n-3+3(n-2)=4 n-9 .
$$

If $g_{i} \in 2 b+\langle a\rangle$, then $g_{i}=2 b+k a$ for some $k \in \mathbb{Z}$ and

$$
\sum(S) \supseteq A_{a} \cup\left(b+B_{a}^{\prime}\right) \cup\left(2 b+C_{a}^{\prime}\right) \cup\left(3 b+k a+B_{a}\right), B_{a} \subseteq B_{a}^{\prime}, C_{a} \subseteq C_{a}^{\prime}
$$

and $A_{a} \cup\left(b+B_{a}^{\prime}\right) \cup\left(2 b+C_{a}^{\prime}\right) \cup\left(3 b+k a+B_{a}\right)$ is a disjoint union, and thus

$$
f(S) \geqslant\left|A_{a}\right|+\left|B_{a}^{\prime}\right|+\left|C_{a}\right|+\left|B_{a}\right| \geqslant n-3+3(n-2)=4 n-9 .
$$

If $g_{i} \in-b+\langle a\rangle$, then $g_{i}=-b+k a$ for some $k \in \mathbb{Z}$ and

$$
\sum(S)=A_{a}^{\prime} \cup\left(b+B_{a}^{\prime}\right) \cup\left(2 b+C_{a}\right) \cup\left(-b+k a+\left(A_{a} \cup\{0\}\right)\right), A_{a} \subseteq A_{a}^{\prime}, B_{a} \subseteq B_{a}^{\prime}
$$

is a disjoint union, and thus

$$
f(S) \geqslant\left|A_{a}\right|+\left|B_{a}\right|+\left|C_{a}\right|+\left|A_{a}\right|+1 \geqslant n-3+3(n-2)=4 n-9 .
$$

If $g_{i} \in-2 b+\langle a\rangle$, then $g_{i}=-2 b+k a$ for some $k \in \mathbb{Z}$ and

$$
\sum(S) \supseteq A_{a}^{\prime} \cup\left(b+B_{a}^{\prime}\right) \cup\left(2 b+C_{a}\right) \cup\left(-b+k a+B_{a}\right), A_{a} \subseteq A_{a}^{\prime}, B_{a} \subseteq B_{a}^{\prime}
$$

is a disjoint union, and thus

$$
f(S) \geqslant\left|A_{a}\right|+\left|B_{a}\right|+\left|C_{a}\right|+\left|B_{a}\right| \geqslant n-3+3(n-2)=4 n-9 .
$$

If $g_{i} \neq g_{j}$ and $g_{i} \neq g_{k}$ and $A \cap B=\emptyset$, then $\sum(S)=A \cup\left\{g_{i}\right\} \cup B, B=\left(g_{i}+A\right)$ is a disjoint union, hence

$$
f(S) \geqslant 2(n-3)+4(n-2)+1 \geqslant 4 n-9 .
$$

Case 4: $\sum\left(S g_{i}^{-1}\right)=A_{a} \cup\left(b+B_{a}\right) \cup\left(-b+C_{a}\right):=A$ for some $1 \leqslant i \leqslant n$. Then $g_{i} \notin\langle a\rangle$ since $\sum(S) \neq A_{a} \cup\left(b+B_{a}\right) \cup\left(-b+C_{a}\right)$. By the definitions of $\sum\left(S g_{i}^{-1}\right)$, we may assume that $S g_{i}^{-1}=\left(S\left(g_{i} g_{j} g_{k}\right)^{-1}\right) g_{j} g_{k}, g_{j}=b+l a \notin\langle a\rangle, g_{k}=-b+l_{1} a \notin\langle a\rangle,\left(S\left(g_{i} g_{j} g_{k}\right)^{-1}\right) \subseteq\langle a\rangle$ and $j \neq k \neq i$. It follows that $\sum\left(S g_{i}^{-1}\right)=\left(\sum\left(S\left(g_{i} g_{j} g_{k}\right)^{-1}\left(l+l_{1}\right) a\right)\right) \cup\left(b+\left(\sum\left(S\left(g_{i} g_{j} g_{k}\right)^{-1}\right) \cup\right.\right.$ $\{0\})) \cup\left(-b+\left(\sum\left(S\left(g_{i} g_{j} g_{k}\right)^{-1}\right) \cup\{0\}\right)\right):=A,\left(\sum\left(S\left(g_{i} g_{j} g_{k}\right)^{-1}\right) \subseteq\langle a\rangle\right.$ is a disjoint union and $\left|S\left(g_{i} g_{j} g_{k}\right)^{-1}\right|=n-3$. And

$$
\sum(S)=A \cup\left\{g_{i}\right\} \cup B, B=\left(g_{i}+A\right) .
$$


The remaining proof of this case is similar to the proof of the case 3, we omit the detail.

Case 5: If the theorem holds for all $S g_{i}^{-1}, 1 \leqslant i \leqslant n$. Let $A=\sum(S) \subseteq G$. Then for any $i$ we have $\sum\left(S g_{i}^{-1}\right) \subseteq\left(A-g_{i}\right) \cap A$, so

$$
\left|\left(A-g_{i}\right) \backslash A\right| \leqslant\left|\sum(S)\right|-\left|\sum\left(S g_{i}^{-1}\right)\right| \leqslant 4 n-10-(4(n-1)-9)=3 .
$$

It is easy to see that $S$ satisfies all the conditions of Lemma 2.9 by the assumptions. Applying Lemma 2.9 to $A \subseteq G$ with generating set $S$, we obtain that either $A$ or $G \backslash A$ has cardinality at most 9 .

We now consider the two cases. If $|G \backslash A|=1$, then $n \leqslant D(G)-1 \leqslant \frac{|G|}{5}+3$, and hence

$$
|G|=|A|+1 \leqslant 4 n-10+1 \leqslant \frac{4}{5}|G|+3 \leqslant|G|-1
$$

since $|G| \geqslant 25$, which is a contradiction.

Otherwise, there is some nonzero element $y \in G \backslash A$, and $S$ is still zero-sum free after appending $-y$, so $n \leqslant D(G)-2 \leqslant \frac{|G|}{5}+2$, and thus

$$
|G| \leqslant|A|+9 \leqslant 4 n-10+9 \leqslant \frac{4}{5}|G|+7 \leqslant|G|-1
$$

when $|G| \geqslant 50$, which is again a contradiction.

The only left case is that $G \cong C_{5} \oplus C_{5}$. If $n=8=D(G)-1$ then $f(S)=24 \geqslant 4 \times 8-9$. The case that $n=7$ follows from [6] Lemma 4.5. The case that $n=6$ follows from the proof of the above case 5 since $f(S)=|A| \geqslant|G|-9 \geqslant 4 \times 6-9$. The case that $n=5$ follows from Theorem 1.1 since $f(S) \geqslant 3 \times 5-4=11=4 \times 5-9$.

\section{Proof of Theorem 1.3:}

Proof. If there exists some integer $i, 1 \leqslant i \leqslant n$ such that the rank of $\left\langle S g_{i}^{-1}\right\rangle$ is two and $f\left(S g_{i}^{-1}\right)=2\left|S g_{i}^{-1}\right|-1$, then by Theorem C we have $S g_{i}^{-1}=a^{x}(a+g)^{y}, a^{x}(a+g)^{y} g, a^{x} b$, where $a, b, g$ are elements of $G$ with $\operatorname{ord}(g)=2$. It follows from our assumption that $g_{i} \notin\left\langle S g_{i}^{-1}\right\rangle$, and thus

$$
f(S)=2 f\left(S x_{i}^{-1}\right)+1=2(2 n-3)+1=4 n-5 .
$$

If $\operatorname{rank}\left\langle S g_{i}^{-1}\right\rangle=2$ and $f\left(S g_{i}^{-1}\right) \geqslant 2\left|S g_{i}^{-1}\right|$, then

$$
f(S)=2 f\left(S g_{i}^{-1}\right)+1=2(2 n-2)+1=4 n-3 .
$$

If $\left\langle S g_{i}^{-1}\right\rangle \cong C_{2} \oplus C_{2} \oplus C_{2 m}$ for some $i, 1 \leqslant i \leqslant n$, then $g_{i} \notin\left\langle S g_{i}^{-1}\right\rangle$ since $\langle S\rangle \not$ $C_{2} \oplus C_{2} \oplus C_{2 m}$, and so

$$
f(S)=2 f\left(S g_{i}^{-1}\right)+1 \geqslant 2(4(n-1)-5)+1=8 n-17>4 n-3
$$

since $n \geqslant 4$, as desired. 
Now we suppose that for all $i, 1 \leqslant i \leqslant n,\left\langle S g_{i}^{-1}\right\rangle$ is an abelian group of rank greater than 2 and $\left\langle S g_{i}^{-1}\right\rangle \neq C_{2} \oplus C_{2} \oplus C_{2 m}$.

First we will show that the theorem holds for $n=4$. Let $S=$ abcd such that $\operatorname{rank}\langle a b c\rangle=\operatorname{rank}\langle a b d\rangle=\operatorname{rank}\langle a c d\rangle=\operatorname{rank}\langle b c d\rangle=3$, then $a, b, c, a+b, a+c, b+c, a+b+c$ are distinct elements in $\sum(a b c d)$ since $\operatorname{rank}\langle a b c\rangle=3$. The case that $\operatorname{rank}\langle a, b, c, d\rangle=4$ is trivial since $f(a b c d)=15$ in this case. It is easy to see that $d \notin\{a, b, c, a+b, a+c, b+c\}$ and $a+d \notin\{a, b, c, a+b, a+c, a+b+c\}$.

(i) If $d=a+b+c, d+a=b+c$ and $d+b=a+c$, then $2 a=2 b=0$ and $\langle S\rangle \cong C_{2} \oplus C_{2} \oplus C_{2 m}$, a contradiction.

(ii) If $d=a+b+c, c=a+b+d$ and $b=a+c+d$, then $2(a+b)=2(a+d)=2(a+c)=0$. Let $b=-a+g_{1}, c=-a+g_{2}, d=-a+g_{3}, o\left(g_{1}\right)=o\left(g_{2}\right)=o\left(g_{3}\right)=2$, then $g_{3}=g_{1}+g_{2}$, and thus $\langle S\rangle \cong C_{2} \oplus C_{2} \oplus C_{2 m}$.

(iii) If $d+a=b+c, d+b=a+c$ and $d+c=a+b$, then $2 a=2 b=2 c=2 d$. Let $b=a+g_{1}, c=a+g_{2}, d=a+g_{3}, o\left(g_{1}\right)=o\left(g_{2}\right)=o\left(g_{3}\right)=2$, then $g_{3}=g_{1}+g_{2}$ and so $\langle S\rangle \cong C_{2} \oplus C_{2} \oplus C_{2 m}$

(iv) If If $d=a+b+c, c=a+b+d$ and $b+a=c+d$, then $2 c=2 d=0,2(a+b)=0$. Let $b=-a+g_{1}, c=g_{2}, d=g_{3}, o\left(g_{1}\right)=o\left(g_{2}\right)=o\left(g_{3}\right)=2$, then $g_{1}=g_{2}+g_{3}$, and thus $\langle S\rangle \cong C_{2} \oplus C_{2} \oplus C_{2 m}$.

By symmetry, we conclude that $\langle S\rangle \cong C_{2} \oplus C_{2} \oplus C_{2 m}$ whenever there are three relations. If there are precisely two relations, then $f(a b c d)=13$; If there is only one relation, then $f(a b c d)=14$; If there is no relations between $a, b, c d$, then $f(a b c d)=15$. Therefore the theorem holds for $n=4$.

Suppose for contradiction that the theorem holds for some abelian group $G$ of minimum size. Choose $S=\left(g_{i}\right)_{i=1}^{n}$ to be a counterexample sequence of minimum length $n \geqslant 5$, so $f(S)<4 n-4$. Also $S$ must generate $G$ by minimality of $|G|, \operatorname{rank}(G)=3$ and $G \nsubseteq C_{2} \oplus C_{2} \oplus C_{2 m}$. Moreover, by the minimality of $n \geqslant 5$, we have that the theorem holds for $S g_{i}^{-1}$.

Let $A=\sum(S) \subset G$, then $\sum\left(S g_{i}^{-1}\right) \subset\left(A-g_{i}\right) \cap A$, and thus $\left|\left(A-g_{i}\right) \backslash A\right| \leqslant|A|-$ $f\left(S g_{i}^{-1}\right) \leqslant 4 n-4-(4 n-7)=3$. It follows from Lemma 2.6 that $\min \{|A|,|G \backslash A|\} \leqslant 5$. Since $|A| \geqslant 2|S|-1 \geqslant 9$, then we have

$$
|G \backslash A| \leqslant 5 .
$$

If $|G \backslash A|=1$, then $n \leqslant D(G)-1 \leqslant \frac{|G|-2}{4}$ by Lemma 2.5 , and hence

$$
|G|=|A|+1 \leqslant 4 n-4+1 \leqslant|G|-5,
$$

is a contradiction. Otherwise, there is some nonzero element $y \in G \backslash A$, and $X$ is still zero-sum-free after appending $-y$, so $n \leqslant D(G)-2 \leqslant \frac{|G|-6}{4}$. Therefore

$$
|G| \leqslant|A|+5 \leqslant 4 n+1 \leqslant|G|-1
$$

is again a contradiction. 


\section{Proof of Theorem 1.4}

Now we are in a position to prove Theorem 1.4.

Proof. If $\operatorname{rank}\langle S\rangle \geqslant 3$, then $f(S) \geqslant 4|S|-5=4\left(n_{r}+2\right)-5 \geqslant 4 n_{r}-1$. If $\operatorname{rank}\langle S\rangle=2$, since $|S|=n_{r}+2 \leqslant D(\langle S\rangle)-1$, then $\langle S\rangle \nRightarrow C_{2} \oplus C_{2 m}, C_{3} \oplus C_{3 m}$. If $\langle S\rangle \cong C_{4} \oplus C_{4 m}$, then $|S|=D(G)-1$ and thus $f(S)=|\langle S\rangle|-1=4 n_{r}-1$. If $\langle S\rangle \neq C_{4} \oplus C_{4 m}$, then $f(S) \geqslant 4|S|-9=4 n_{r}-1$ by Theorem 1.2. We are done.

Similarly, by Theorem 1.1, we can prove the following theorem in [6].

Theorem 4.1 ([6] Theorem 1.1) Let $G=C_{n_{1}} \oplus \ldots \oplus C_{n_{r}}$ be a finite abelian group with $1<n_{1}|\ldots| n_{r}$. If $r \geqslant 2$ and $n_{r-1} \geqslant 3$, then every zero-sum free sequence $S$ over $G$ of length $|S|=n_{r}+1$ satisfies $f(S) \geqslant 3 n_{r}-1$.

Proof. If $\operatorname{rank}\langle S\rangle \geqslant 3$, then $f(S) \geqslant 4|S|-5=4\left(n_{r}+1\right)-5 \geqslant 3 n_{r}-1$. If $\operatorname{rank}\langle S\rangle=2$, since $|S|=n_{r}+1 \leqslant D(\langle S\rangle)-1$, then $\langle S\rangle \not C_{2} \oplus C_{2 m}$. Therefore $f(S) \geqslant 3|S|-4 \geqslant$ $3\left(n_{r}+1\right)-4=3 n_{r}-1$ by Theorem 1.1. We are done.

We recall a conjecture by Bollobás and Leader, stated in [2].

Conjecture 4.1 Let $G=C_{n} \oplus C_{n}$ with $n \geqslant 2$ and let $\left(e_{1}, e_{2}\right)$ be a basis of $G$. If $k \in[0, n-2]$ and

$$
S=e_{1}^{n-1} e_{2}^{k+1} \in \mathcal{F}(G) .
$$

Then we have $f(G, n+k)=f(S)=(k+2) n-1$.

By a main result of [6] and Theorem 1.4, the conjecture holds for $k \in\{0,1,2, n-2\}$. Moreover, the following general conjecture stated in [6] holds for $k=2$.

Conjecture 4.2 Let $G=C_{n_{1}} \oplus \ldots \oplus C_{n_{r}}$ be a finite abelian group with $r \geqslant 2$ and $1<n_{1}|\ldots| n_{r}$. Let $\left(e_{1}, \ldots, e_{r}\right)$ be a basis of $G$ with ord $\left(e_{i}\right)=n_{i}$ for all $i \in[1, r], k \in$ $\left[0, n_{r-1}-2\right]$ and

$$
S=e_{r}^{n_{r}-1} e_{r-1}^{k+1} \in \mathcal{F}(G) .
$$

Then we have $f\left(G, n_{r}+k\right)=f(S)=(k+2) n_{r}-1$.

\section{References}

[1] J.D. Bovey, P. Erdős, and I. Niven, Conditions for zero sum modulo n, Canad. Math. Bull. 18 (1975), $27-29$.

[2] B. Bollobás and I. Leader, The number of k-sums modulo k, J. Number Theory 78(1999), 27-35.

[3] S.T. Chapman and W.W. Smith, A characterization of minimal zero-sequences of index one in finite cyclic groups, Integers 5(1) (2005), Paper A27, 5pp. 
[4] R.B. Eggleton and P. Erdős, Two combinatorial problems in group theory, Acta Arith. 21(1972), 111-116.

[5] W.D. Gao and I. Leader, sums and $k$-sums in an abelian groups of order $k$, J. Number Theory 120(2006), 26-32.

[6] W. Gao, Y. Li, J. Peng and F. Sun, On subsequence sums of a zero-sum free sequence II, The Electronic Journal of Combinatorics, 15(2008), $\sharp$ R117.

[7] A. Geroldinger and F. Halter-Koch, Non-Unique Factorizations. Algebraic, Combinatorial and Analytic Theory, Pure and Applied Mathematics, Vol. 278, Chapman \& Hall/CRC, 2006.

[8] O. Ordaz and D. Quiroz, The Erdös-Ginzburg-Ziv theorem in abelian non-cyclic groups Divulg. Mat. 8(2)(2000)113-119.

[9] J. E. Olson and E.T.White, sums from a sequence of group elements, in : Number Theory and Algebra, Academic Press, New York, 1977, pp. 215-222.

[10] A. Pixton, sSequences with small subsums sets, J. Number Theory 129(2009), 806817.

[11] F. Sun, On subsequence sums of a zero-sum free sequence, The Electronic Journal of Combinatorics. 14(2007), $\sharp \mathrm{R} 52$.

[12] P.Z. Yuan, On the index of minimal zero-sum sequences over finite cyclic groups, J. Combin. Theory Ser. A 114(2007), 1545-1551.

[13] P.Z. Yuan, Subsequence sums of a zero-sumfree sequence, European Journal of Combinatorics, 30(2009), 439-446. 